\title{
A novel function of p53 in TR3-MDM2 cross-talk
}

\author{
Hang-Zi Chen ${ }^{1}$, Qiao $\mathrm{Wu}^{1}$ \\ ${ }^{1}$ Key Laboratory of the Ministry of Education for Cell Biology and Tumor Cell Engineering, School of Life Sciences, Xiamen University, \\ Fujian 361005, China
}

Cell Research (2007) 17:293-294. doi: 10.1038/cr.2007.23; published online 10 April 2007

TR3 (also known as Nur77, or NGFI-B) belongs to the steroid/thyroid/retinoid receptor superfamily, and is classified as an orphan receptor because its specific ligand has not been identified [1]. Originally, TR3 was identified as a growth factor-inducible gene [2]. The role of TR3 in apoptosis was first reported in 1994, where TR3 expression was shown to be induced by T-cell receptor signaling and required for T-cell receptor-mediated apoptosis $[3,4]$. Rapid induction of TR3 is also observed in different types of cancer cells after stimulation by apoptosis-inducing agents [5-7], indicating that induction of TR3 contributes to the apoptotic process.

TR3 also functions as a transcription factor which positively or negatively regulates gene expression. Our previous study revealed that TR3 heterodimerizes with the retinoid X receptor $\alpha(\mathrm{RXR} \alpha)$, and binds to the retinoic acid response element in the retinoic acid receptor $\beta$ (RAR $\beta$ ) promoter, thereby promoting RAR $\beta$ expression which contributes to apoptosis induction in breast cancer cells [8]. TR3 also heterodimerizes with another orphan receptor, COUP-TF, to regulate the expression of RAR $\beta$ in lung cancer cells [9]. In this commentary, we tell another story about how TR3 inhibits MDM2 gene and protein expression through the mediation of $\mathrm{p} 53$.

A study from Lee's group suggested that MDM2 was another possible target of TR3, as TR3-induced decrement of MDM2 expression contributed to the stabilization of hypoxia-inducible factor- $1 \alpha[10]$. However, we did not detect any direct interaction between TR 3 and MDM 2 in vivo and in vitro, suggesting that another factor might be involved

\footnotetext{
Correspondence: Qiao Wu

Tel: +86-592-2187959; Fax: +86-592-2086630

E-mail: qiaow@xmu.edu.cn
}

in the TR3-MDM2 cross-talk. We found that 553 functions as a linking platform through interaction with both TR3 and MDM2, which allows TR3 to inhibit the expression of MDM2 at both transcriptional and post-transcriptional levels. On one hand, binding of TR3 to p53 leads to the repression of p53 transcriptional activity by inhibiting p300-induced acetylation of p53, which accounts for the inhibitory effect of TR3 on the transcriptional activity of the MDM2 promoter. On the other hand, binding of TR3 to 553 sequestrates p53 away from MDM2. As a result, TR3 accelerates MDM2 protein auto-ubiquitination and degradation. These findings suggest that when the activity of p53 is kept at high levels, e.g. after DNA damage, the presence of TR3 would result in "super-inhibition" of MDM2 and "super-induction" of p53, thus significantly enhancing the biological function of $\mathrm{p} 53$. Consistent with this notion, we also found that, dependent on the activity of $\mathrm{p} 53$, over-expression of TR3 sensitized hepatoma cells to UV-induced apoptosis, although TR3 repressed p53-dependent transcription of the 121 wafl/cipl gene. Therefore, the physiological levels of $\mathrm{p} 53$ protein may be important in setting the threshold for TR3-mediated apoptosis.

In summary, this work demonstrates a novel mechanism whereby TR3 regulates MDM2 through p53 mediation at both transcriptional and post-transcriptional levels (Figure 1). Notably, the TR3-p53 interaction not only reduces MDM2 expression, but also protects p53 from MDM2mediated degradation. Since destabilization of MDM2 and stabilization of p53 are important approaches for cancer therapy, the current study reveals that TR3 may be a novel target for the development of new anticancer strategy that may restrict MDM2-induced tumor progression. In addition, whether and how the interaction between p 53 and TR3 can be further exploited for better therapeutic measures in oncology warrants further investigations. 


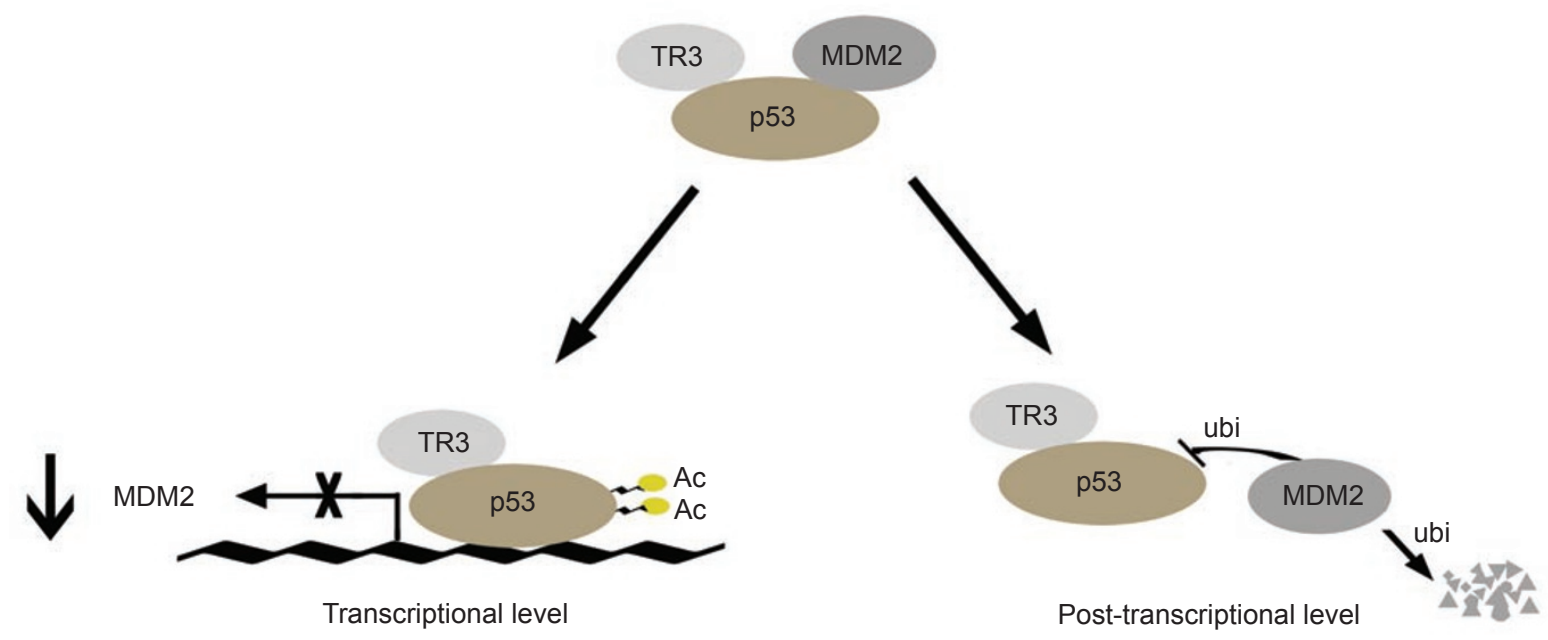

Figure 1 A novel model for regulation of MDM2 by TR3 through p53 mediation at both transcriptional and post-transcriptional levels.

\section{References}

1. Chang C, Kokontis J. Identification of a new member of the steroid receptor super-family by cloning and sequence analysis. Biochem Biophys Res Commun 1988; 155:971-977.

2. Hazel TG, Nathans D, Lau LF. A gene inducible by serum growth factors encodes a member of the steroid and thyroid hormone receptor superfamily. Proc Natl Acad Sci USA 1988; 85:84448448 .

3. Liu ZG, Smith SW, McLaughlin KA, Schwartz LM, Osborne BA. Apoptotic signals delivered through the T-cell receptor of a T-cell hybrid require the immediate-early gene nur77. Nature 1994; 367:281-284.

4. Woronicz JD, Calnan B, Ngo V, Winoto A. Requirement for the orphan steroid receptor Nur77 in apoptosis of T-cell hybridomas. Nature 1994; 367:277-281.

5. Li H, Kolluri SK, Gu J, et al. Cytochrome $c$ release and apoptosis induced by mitochondrial targeting of nuclear orphan receptor TR3. Science 2000; 289:1159-1164.

6. Li Y, Lin B, Agadir A, et al. Molecular determinants of AHPN (CD437)-induced growth arrest and apoptosis in human lung cancer cell lines. Mol Cell Biol 1998; 18:4719-4731.

7. Wu Q, Liu S, Ye XF, Huang ZW, Su WJ. Dual roles of Nur77 in selective regulation of apoptosis and cell cycle by TPA and ATRA in gastric cancer cells. Carcinogenesis 2002; 23:1583-1592.

8. Wu Q, Dawson MI, Zheng Y, et al. Inhibition of trans-retinoic acid-resistant human breast cancer cell growth by retinoid $\mathrm{X}$ receptor-selective retinoids. Mol Cell Biol 1997; 17:6598-6608.

9. Wu Q, Li Y, Liu R, et al. Modulation of retinoic acid sensitivity in lung cancer cells through dynamic balance of orphan receptors nur77 and COUP-TF and their heterodimerization. EMBO J 1997; 16:1656-1669.

10. Yoo YG, Yeo MG, Kim DK, Park H, Lee MO. Novel function of orphan nuclear receptor Nur77 in stabilizing hypoxia-inducible factor-1alpha. J Biol Chem 2004; 279:53365-53373. 